\title{
Cardiomyocyte-restricted high-mobility group box 1 (HMGB1) deletion leads to small heart and glycolipid metabolic disorder through GR/PGC-1a signalling
}

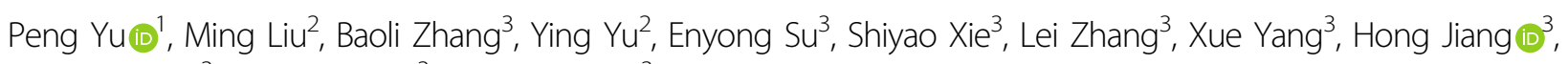
Ruizhen Chen ${ }^{3}$, Yunzeng Zou ${ }^{3}$ and Junbo $\mathrm{Ge}^{3}$

\begin{abstract}
Cardiac growth and remodelling are key biological processes influencing the physiological performance of the heart, and a previous study showed a critical role for intracellular HMGB1 in vitro. However, the in vivo study, which used conditional Hmgb1 ablation, did not show a significant effect on cellular or organic function. We have demonstrated the extracellular effect of HMGB1 as a pro-inflammatory molecule on cardiac remodelling. In this study, we found that HMGB1 deletion by cTnT-Cre in mouse hearts altered glucocorticoid receptor (GR) function and glycolipid metabolism, eventually leading to growth retardation, small heart and heart failure. The subcellular morphology did not show a significant change caused by HMGB1 knockout. The heart showed significant elevation of glycolysis, free fatty acid deposition and related enzyme changes. Transcriptomic analysis revealed a list of differentially expressed genes that coincide with glucocorticoid receptor function in neonatal mice and a significant increase in inflammatory genes in adult mice. Cardiac HMGB1 knockout led to a series of changes in PGC-1a, UCP3 and GyK, which were the cause of metabolic changes and further impacted cardiac function. Ckmm-Cre Hmgb1 $1^{\mathrm{fl} / \mathrm{ll}}$ mice did not show a specific phenotype, which was consistent with the reported negative result of cardiomyocyte-specific Hmgb1 deletion via MHC-Cre. We concluded that HMGB1 plays essential roles in maintaining normal cardiac growth, and different phenotype from cardiac-specific HMGB1-deficient mice may be caused by the cross with mice of different Cre strains.
\end{abstract}

\section{Introduction}

Cardiac growth and remodelling are key biological processes influencing the physiological performance of the heart. Cardiomyopathy is one of the most common causes of heart failure and is characterized by cardiac remodelling and contractile dysfunction. However, the molecular mechanisms underlying the pathophysiology of

\footnotetext{
Correspondence: Hong Jiang (jianghong_@fudan.edu.cn)

'Department of Endocrinology and Metabolism, Fudan Institute of Metabolic Diseases, Zhongshan Hospital, Fudan University, Shanghai, China

Department of General Practice, Zhongshan Hospital, Shanghai Medical College of Fudan University, Shanghai, China

Full list of author information is available at the end of the article

These authors contributed equally: Peng Yu, Ming Liu

Edited by Alessandro Rufini
}

decompensated heart failure remain largely unknown. Strategies targeting new molecules or factors are urgently needed to prevent this transition under pathological conditions ${ }^{1}$.

High-mobility group box 1 (HMGB1), which exhibits diverse biological functions depending on cellular location, is a non-histone DNA-binding nuclear protein present in nearly all cell types ${ }^{2}$. Intracellular HMGB1 regulates autophagy, facilitates DNA repair and regulates transcriptional regulation ${ }^{3-5}$. However, in vivo validation of the versatile functions of HMGB1 shows its dispensable role in adult organisms ${ }^{6}$. Furthermore, newborn Hmgb1deficient mice exhibited lethal hypoglycaemia ${ }^{7,8}$. Mice of different Cre strains could lead to diverse phenotypes

\section{(c) The Author(s) 2020}

(c) (i) Open Access This article is licensed under a Creative Commons Attribution 4.0 International License, which permits use, sharing, adaptation, distribution and reproduction cc. in any medium or format, as long as you give appropriate credit to the original author(s) and the source, provide a link to the Creative Commons license, and indicate if changes were made. The images or other third party material in this article are included in the article's Creative Commons license, unless indicated otherwise in a credit line to the material. If material is not included in the article's Creative Commons license and your intended use is not permitted by statutory regulation or exceeds the permitted use, you will need to obtain permission directly from the copyright holder. To view a copy of this license, visit http://creativecommons.org/licenses/by/4.0/. 
resulting from asynchronous functional loss of the floxed genes, which may reveal more detailed molecular roles in biological processes ${ }^{9}$.

This work focused on the role of HMGB1 in the development of the heart by conditional cardiac-specific HMGB1 knockout using cTnT-Cre/+ mice, in which the target loxP sites could recombine starting from E7.5, resulting in earlier and more uniform recombination in the developing heart than that induced by Myh6-Cre ${ }^{10-12}$. We found that cardiac-specific knockout of HMGB1 resulted in small and dysfunctional hearts and the underlying mechanisms of glucocorticoid receptors (GRs) and metabolic changes. Our data showed that HMGB1 plays a vital role in shaping cardiac function.

\section{Results}

\section{Generation of cardiac HMGB1 knockout mice}

To investigate the roles of HMGB1 in cardiomyocytes in vivo, we generated cardiac-specific HMGB1 knockout mice using Cre-loxP technology. The mouse breeding strategy to generate cardiac-specific HMGB1 knockout mice is shown in Fig. 1a. Gel electrophoresis of PCR genotyping of Cre and Hmgb1-loxp is shown in Fig. S1a and $1 \mathrm{~b}$ separately. Moreover, verification of the HMGB1 knockout was demonstrated by reverse transcriptase PCR (RT-PCR) with primers specific to exons 2-4 of HMGB1 (Fig. 1b). HMGB1 knockout was confirmed by western blotting demonstrating that both neonatal and 12-week-old Hmgb1 $\triangle \mathrm{CM}$ mice had a significant decrease in HMGB1. (Fig. 1c) The expression of HMGB1 in heart tissue is reduced, but not abolished. The residual HMGB1 may result from stromal cells without cardiac Troponin $\mathrm{T}$ (cTnT) expression. We further exclude the leakage of the cTnT-Cre mice using western blot to detect HMGB1 in the liver, spleen, lung, kidney, muscle, ovary and testis, which showed no difference between the two groups (Fig. S1e). Immunofluorescence (IF) staining in frozen cardiac sections from neonatal and adult mice revealed undetectable HMGB1 expression in the Hmgb1 $\triangle \mathrm{CM}$ group compared to the $\mathrm{Hmgb}^{\mathrm{f} / \mathrm{fl}}$ group (Fig. 1d, e).

\section{Genetic deletion of HMGB1 in cardiomyocytes results in growth retardation and small hearts}

Hmgb1 $\triangle \mathrm{CM}$ showed significant retardation of body growth (Fig. 2a). The female mice were infertile, while the males were fertile after 16 weeks of age, in comparison with the $\mathrm{HMGB1}^{\mathrm{fl} / \mathrm{fl}}$ mice, which could reproduce from 6-8 weeks old. We detected HMGB1 in ovary and testis to exclude the work of Cre in gonads which showed no difference (Fig. S1e). The littermates were born with similar appearance and body weight (Fig. S1c), whereas the $\mathrm{Hmgb1}^{\triangle \mathrm{CM}}$ littermates showed significantly decreased body mass (Fig. S1d).
We suspected that the retardation of body growth was caused by cardiac deficiency. We found that the gross appearance of $\mathrm{Hmgb}_{1} \triangle \mathrm{CM}$ hearts was diminished (Fig. 2b) and that heart weight/tibial length ratio (HW/ $\mathrm{TL}$ ) and heart weight/body weight ratio (HW/BW) were decreased significantly (Fig. 2c and Fig. S2a). No structural abnormalities were observed by haematoxylin and eosin (H\&E) staining (Fig. 2d), and there were also no significant structural differences in the neonatal heart (Fig. S2b). Furthermore, Sirius red staining demonstrated that the degree of fibrosis in $\mathrm{Hmgb1}^{\triangle \mathrm{CM}}$ hearts was more severe (Fig. 2e). Wheat germ agglutinin (WGA) staining demonstrated no significant variance in the cell surface between the two groups (Fig. 2f, g). To detect the cause of small heart size, we performed staining with Ki-67, a cellular marker for proliferation, which was significantly decreased in neonatal Hmgb1 $\triangle \mathrm{CM}$ mice (Fig. 2h, i). Additionally, Hmgb1 $\triangle \mathrm{CM}$ inhibited myocardial expression of canonical hypertrophic marker genes (Fig. 2j), and transmission electron microscopy (TEM) showed no mitochondrial structural changes in the Hmgb1 $\triangle \mathrm{CM}$ mice (Fig. S2c).

\section{Cardiac HMGB1 knockout mice developed cardiac dysfunction}

We further performed functional and morphological examinations of the heart. Echocardiographic measurements indicated compromised cardiac function (Fig. 3a). In addition to the thinner ventricular wall and smaller ventricular volume (Fig. $3 \mathrm{~b}, \mathrm{c}$ ), the $\mathrm{Hmgb}{ }^{\triangle} \triangle \mathrm{CM}$ mice showed reduced left ventricular ejection fraction (LVEF) and fractional shortening (Table 1). Haemodynamic parameters showed that HMGB1-cKO induced a significant decrease in LV peak systolic pressure (LVP sys, $99 \mathrm{mmHg}$ ) compared to the HMGB1 ${ }^{\mathrm{fl} / \mathrm{fl}}$ condition (131 mmHg) (Fig. 3d). Moreover, both $\mathrm{d} P / \mathrm{d} t \max$ (peak rate of ventricular pressure rise) and $\mathrm{d} P / \mathrm{d} t$ min (peak rate of ventricular pressure decline) were decreased significantly by cardiac-specific knockout of HMGB1 (Fig. 3e).

\section{Cardiac HMGB1 knockout changed glucose and lipid metabolism}

As HMGB1 ${ }^{-1-}$ mice have been reported to die because of severe hypoglycaemia ${ }^{8}$, we next analysed the blood glucose levels of newborns. At birth, the level of blood glucose showed a decreasing trend in the Hmgb1 $\triangle \mathrm{CM}$ siblings. The random blood glucose measurements were similar for the adult mice. However, cardiac-specific HMGB1 knockout led to a significant decrease in fasting blood glucose (Fig. 4a). To further prove the HMGB1dependent reconfiguration of energy metabolism, we performed an [18F]-fluorodeoxyglucose positron emission tomographic analysis in living animals to unambiguously 


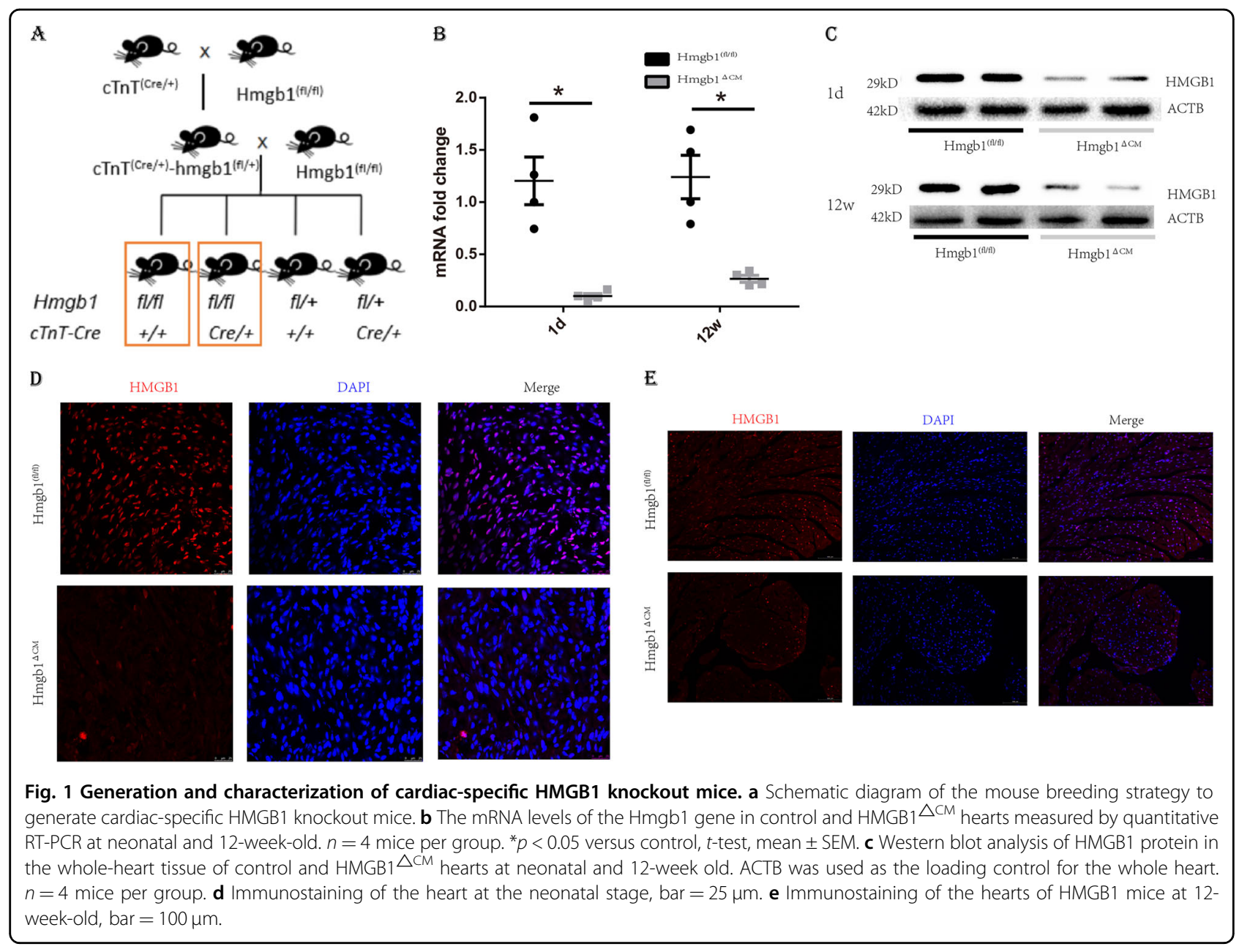

demonstrate that HMGB1 affects myocardial metabolism in the beating heart. Our measurements revealed a significant increase in glucose uptake and glycolytic flux in the heart after deletion of HMGB1 in adult cardiomyocytes (Fig. 4b, c).

We further detected the total fatty acids and free fatty acids, and the total fatty acids between the two groups showed little difference (Fig. 4d). However, the cardiacspecific knockout of HMGB1 led to a significant elevation of free fatty acids (Fig. 4e), which might be caused by a disturbance in cardiac metabolism. We detected the enzymes involved in glucose and free fatty acid metabolism. The results showed an increase in the enzymes facilitating glycolysis, including GLUT1, GLUT4, HK and $\mathrm{PDH}$, and a decrease in CPT1, a key enzyme involved in the utilization of free fatty acids (Fig. 4f).

\section{Cardiac HMGB1 knockout represses the glucocorticoid response}

To gain further mechanistic insights into the function of HMGB1 in the heart, we performed RNA-sequencing analysis of hearts from $\mathrm{Hmgb}_{1} \triangle \mathrm{CM}$ and $\mathrm{Hmgb1}^{\mathrm{f} / \mathrm{fl}}$ mice.
The genes detected was shown in Tables S2 and S3. As expected, we detected numerous deregulated genes that reflect pathological changes in HMGB1-deficient hearts (Fig. 5a, c). More interestingly, gene ontology term analysis in Hmgb1 $\triangle \mathrm{CM}$ hearts using the Database for Annotation, Visualization and Integrated Discovery (DAVID) revealed significant changes in genes involved in cell growth and response to glucocorticoids as well as changes in genes involved in metabolic processes (Fig. 5b, d). We hypothesized that HMGB1 knockout suppresses glucocorticoid pathways, further resulting in the adult phenotype, which includes metabolic changes and inflammatory activation.

Glucocorticoids work through multiple mechanisms to bring about their desired effects ${ }^{13}$. HMGB1 has been implied to be a cofactor to facilitate the effects of $\mathrm{GRs}^{3,8,14}$. We therefore reasoned that HMGB1 physically interacts with GRs and prepared WT mouse heart tissue lysates from neonatal, 2-week-old and 12-week-old mice for immunoprecipitation using anti-HMGB1 or GR antibodies. HMGB1 pulled down GR, and reciprocal immunoprecipitation of GR pulled down HMGB1 (Fig. 5e). 


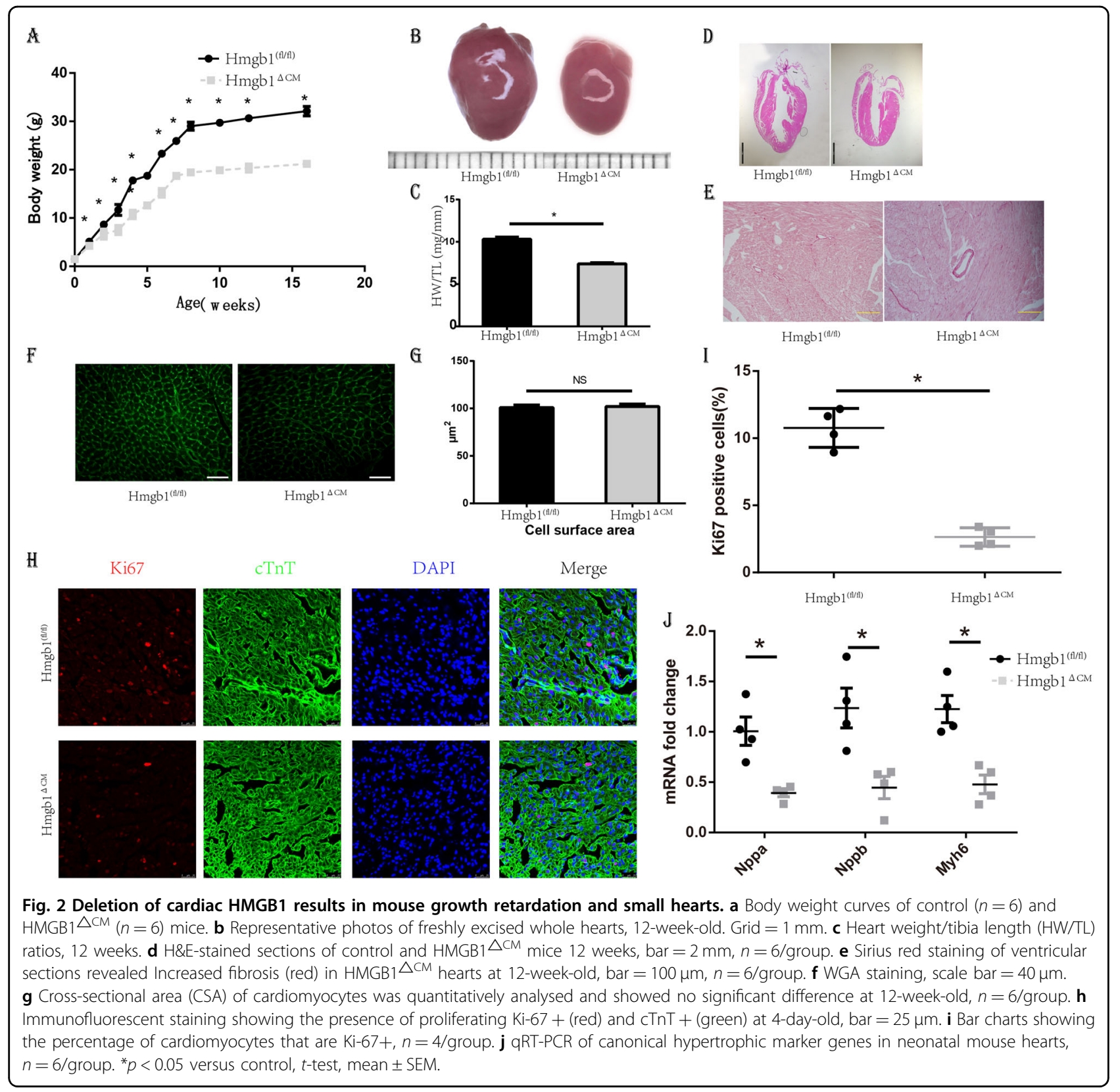

Interestingly, Agresti et al. reported that HMGB1 facilitated GR binding with chromatin. Therefore, we performed chromatin immunoprecipitation (ChIP) in WT and Hmgb1 $\triangle \mathrm{CM}$ neonatal mice, which showed that a lack of HMGB1 resulted in clearly diminished binding of GR to the genomic loci of Fkbp5 and Scn9a reported to be the GR-binding target (Fig. 5f) ${ }^{15}$. We further detected the downstream genes of GR from neonatal heart tissue ${ }^{16}$, and found the Pdk4, Arrdc3, Arrdc2, Abra, Klf15 and Nfkbia downregulated, Cxcr4 upregulated (Fig. 5g). The genes were related with glucose/lipid metabolism or inflammatory response either.
HMGB1 mainly functions as an inflammatory mediator, and glucocorticoids are used as anti-inflammatory therapy. The transcriptome of the adult heart showed that inflammatory genes were elevated significantly in 12week-old mice. Therefore, the metabolic changes in $\mathrm{Hmgb}^{\triangle \mathrm{CM}}$ mice may indicate a sequence of inflammation or an effect of GR dysfunction.

\section{The cardiac metabolic change was caused by GR-PGC-1a signalling}

Physiological glucocorticoid levels improve the contractility of primary-mouse-foetal cardiomyocytes by 


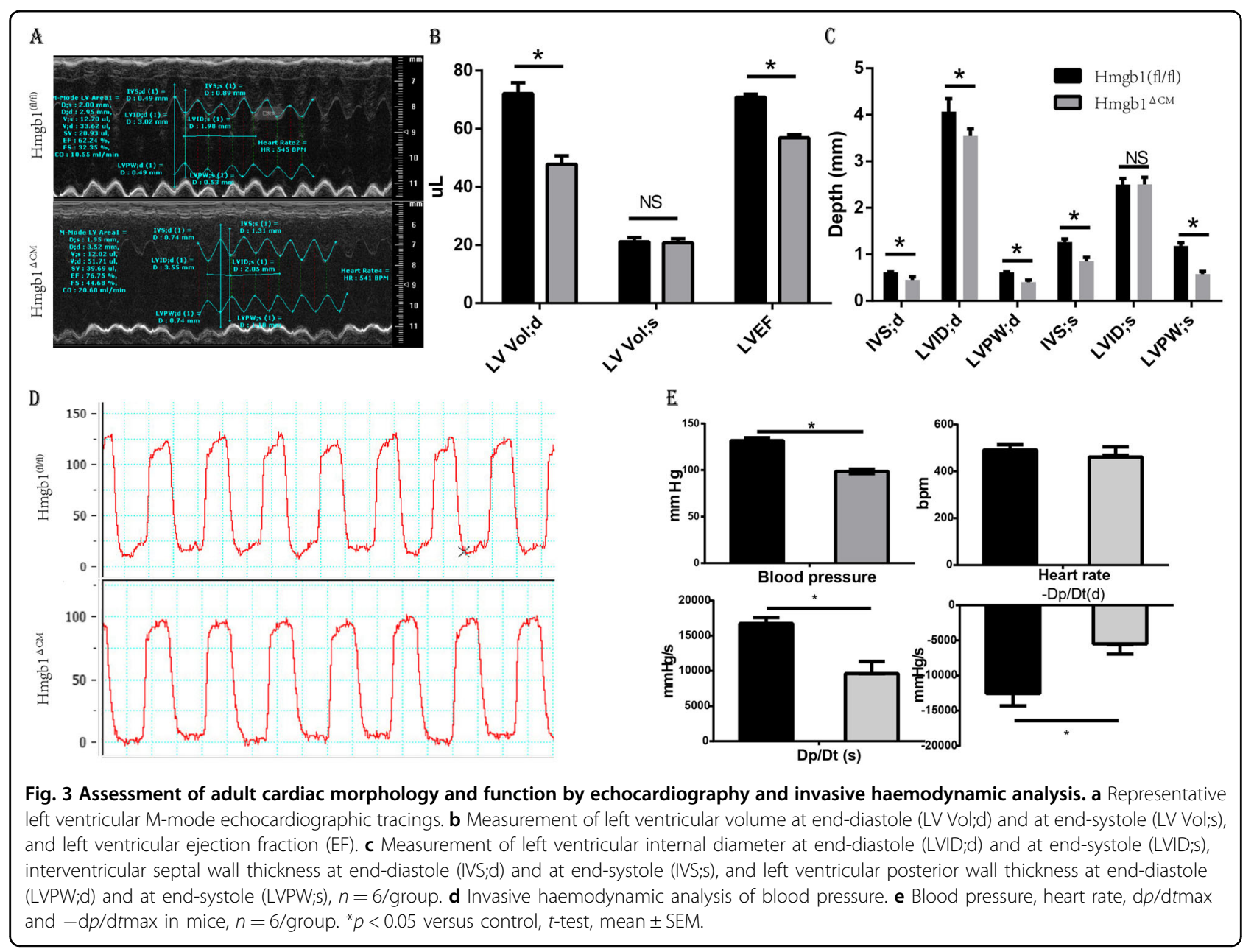

inducing PGC-1 $\alpha$, a key mediator of glucocorticoidinduced maturation of cardiomyocytes ${ }^{17}$. Therefore, we detected PGC- $1 \alpha$ and found downregulation of PGC- $1 \alpha$ in $\mathrm{Hmgb1}^{\triangle \mathrm{CM}}$ mice. The downregulation of PGC-1 $\alpha$ could impair fatty acid oxidation ${ }^{18}$ and increase glucose utilization $^{19}$. UCP3 was also elevated in the heart and was postulated to export fatty acid anions from the mitochondrial matrix when fatty acyl-CoA levels were increased (Fig. 6a) ${ }^{20}$. Moreover, glycerol is a substrate for cardiac energy production and ${ }^{21}$ the glycerol kinase (GyK) could facilitate the glycerol oxidation and affect the deposition of fatty acid. We detected the GyK and found it decreased in the Hmgb1 $\triangle \mathrm{CM}$ groups, which in line with the report that GyK deficiency cause growth retardation and fat metabolism alteration, as a downstream of PGC$1 \alpha^{22,23}$. Therefore, we proposed that HMGB1 deficiency may cause lipid deposition. Oil red $\mathrm{O}$ staining showed that HMGB1 knockout led to lipid deposition in the myocardium (Fig. 6b), which coincides with a recent study showing that ablation of Hmgb1 in intestinal epithelial cells causes intestinal lipid accumulation ${ }^{24}$. We further performed dihydroethidium staining (Fig. 6c) in the control and $\mathrm{Hmgb1}^{\triangle \mathrm{CM}}$ groups, which revealed that HMGB1 knockout resulted in elevated levels of ROS.

\section{Discussion}

In our present study, we used a cardiac-specific cTnTCre system to delete HMGB1 in cardiomyocytes. We found that deletion of HMGB1 led to impaired heart growth and left ventricular dysfunction, accompanied by a dramatic decrease in body weight. This finding demonstrated that HMGB1 deletion in cardiomyocytes resulted in cardiomyopathy, which further led to heart failure and impaired body growth. Although knockout experiments using $\alpha$ MHC-Cre have shown that HMGB1 is dispensable for the heart ${ }^{25}$, the in vitro functions of HMGB1 have been demonstrated in mitochondrial quality control $^{26}$. Our present study provided evidence for the first time, to our knowledge, that HMGB1 plays an essential role in maintaining normal cardiac growth and function in mice. On the other hand, deletion of HMGB1 in mouse cardiomyocytes was also associated with low blood glucose, 
Table 1 Echocardiographic parameters of the two groups.

\begin{tabular}{llll}
\hline & Hmgb1 (fl/fl) & Hmgb1 $\triangle$ cm & $p$ value \\
\hline IVS;d & $0.61 \pm 0.022$ & $0.49 \pm 0.014$ & 0.0001 \\
LVID;d & $4.037 \pm 0.092$ & $3.393 \pm 0.088$ & 0.0001 \\
LVPW;d & $0.621 \pm 0.019$ & $0.465 \pm 0.015$ & 0.0001 \\
IVS;S & $1.232 \pm 0.029$ & $0.854 \pm 0.017$ & 0.0001 \\
LVID;S & $2.431 \pm 0.071$ & $2.4 \pm 0.071$ & 0.77 \\
LVPW;S & $1.146 \pm 0.029$ & $0.68 \pm 0.036$ & 0.0001 \\
LV Vol;d & $72.01 \pm 3.80$ & $47.80 \pm 2.89$ & 0.0001 \\
LV Vol;s & $21.09 \pm 1.50$ & $20.74 \pm 1.44$ & 0.87 \\
\%EF & $70.86 \pm 1.07$ & $56.93 \pm 1.09$ & 0.0001 \\
\% FS & $39.83 \pm 0.90$ & $29.08 \pm 0.70$ & 0.0001 \\
Heart rate & $521.4 \pm 5.37$ & $508.8 \pm 5.46$ & 0.13 \\
\hline
\end{tabular}

IVS; $d$ interventricular septal wall thickness at end-diastole, IVS; interventricular septal wall thickness at end-systole, LVID; $d$ left ventricular internal diameter at end-diastole, $L V I D ; s$ left ventricular internal diameterat end-systole, $L V P W ; d$ left ventricular posterior wall thickness at end-diastole, $L V P W ;$ s left ventricular posterior wall thickness at end-systole, LV Vol;d left ventricular volume at enddiastole, $L V$ Vol;s left ventricular volume at end-systole, $L V E F$ left ventricular ejection fraction, FS fractional shortening. and changes in the genes associated with inflammation and GRs were also consistent with previous studies showing that HMGB1 was associated with GR function ${ }^{8}$.

HMGB1 was first found to be a non-histone nuclear protein, facilitating nucleosome structure and enhancing DNA repair and chromatin modification ${ }^{27}$. Overexpression of HMGB1 could prevent DNA damage induced by pressure overload ${ }^{5}$. It was also reported to be essential for mitochondrial quality control or to facilitate mitochondrial restoration ${ }^{28,29}$. Previously, we showed that HMGB1 was involved in cardiac remodelling ${ }^{5,30}$. To investigate the effect of intracellular HMGB1 in the myocardium, we constructed cardiac conditional HMGB1 knockout mice and found that a lack of HMGB1 retarded heart growth and impaired its function, which may be caused by the effect on GR/PGC- $1 \alpha$ and imbalance of glucose and fatty acid metabolism.

In our present study, we also performed RNAsequencing analysis to identify the effects of HMGB1 deletion on cardiac gene homoeostasis. In the neonatal heart, there were 112 upregulated and 74 downregulated genes in the Hmgb1 $\triangle \mathrm{CM}$ hearts relative to their control counterparts. Most of these genes are related to cell

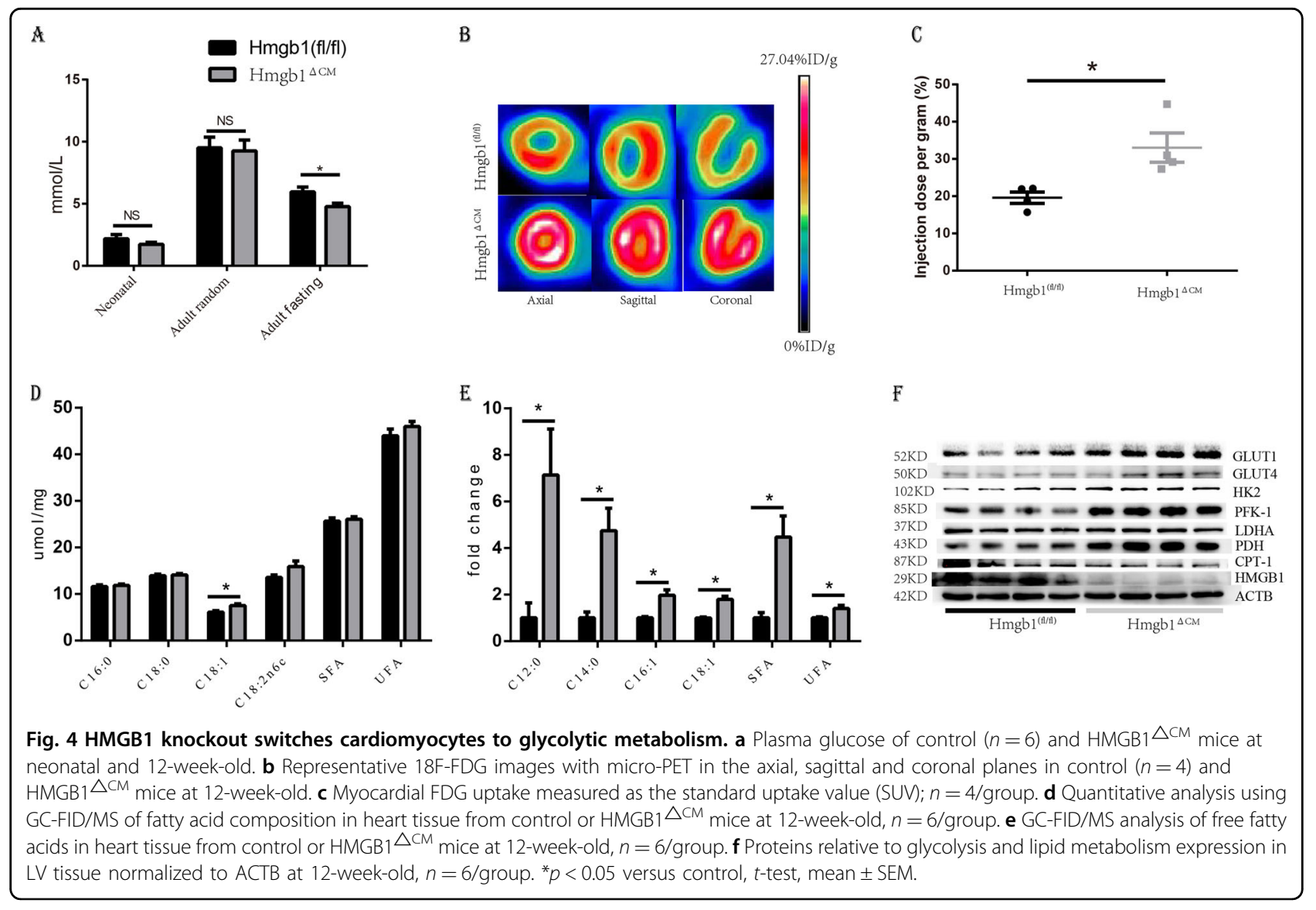




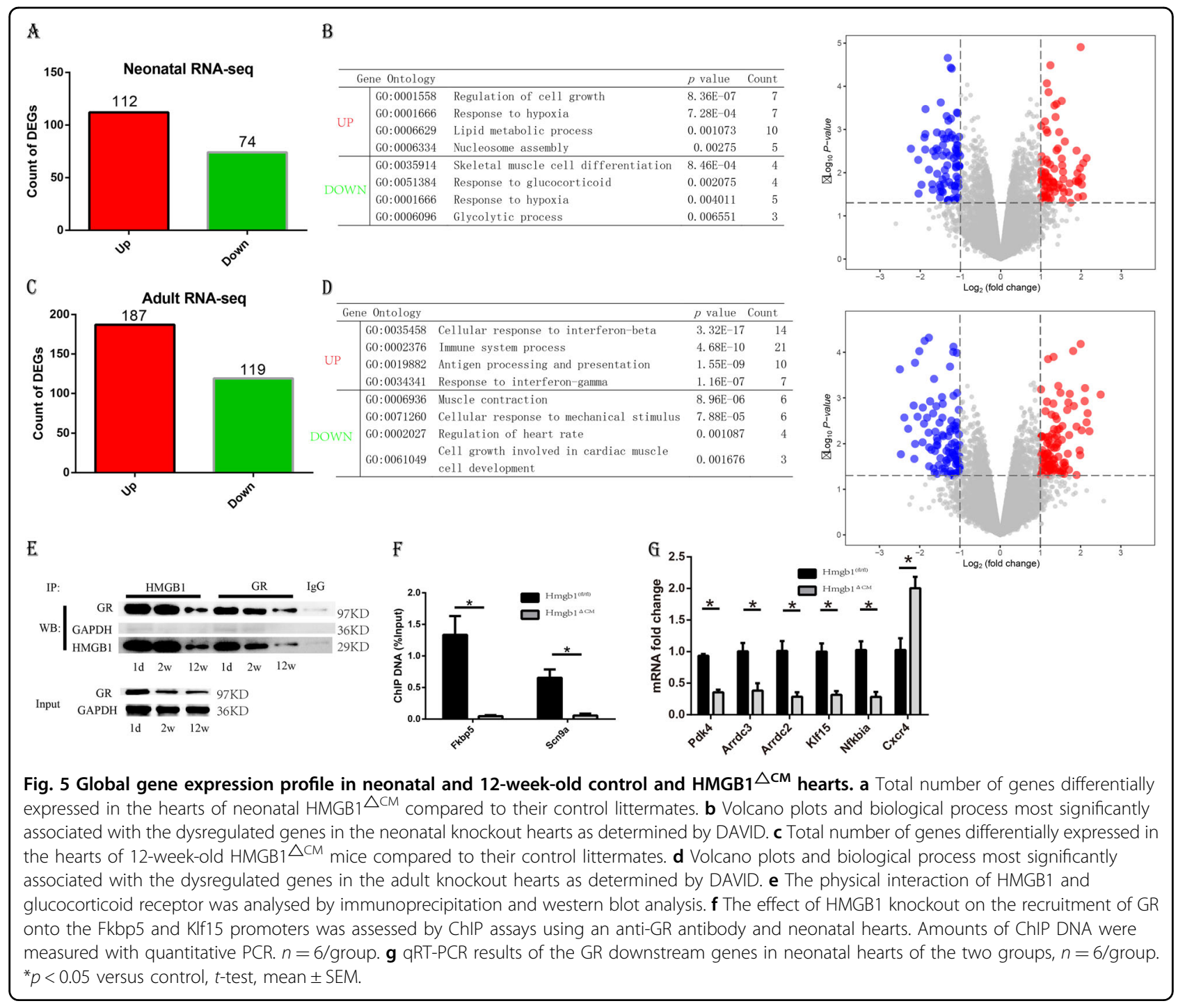

growth, muscle differentiation and the response to glucocorticoids, which further indicates the influence of genes involved in lipid metabolic changes. Therefore, we provided for the first time, at least to our knowledge, a list of genes affected by HMGB1-mediated neonatal mouse cardiomyocytes. In the adult mouse, HMGB1 knockout led to 187 upregulated and 119 downregulated genes. Interestingly, the upregulated genes are mostly related to the inflammatory process, in contrast with the opinion that HMGB1 acts as a pro-inflammatory protein. The downregulated genes are mostly related to cell muscle contraction or growth.

Conditional cardiac knockout of HMGB1 first led to decreased expression of cardiac foetal genes, including $\mathrm{ANF}$ and $\beta \mathrm{MHC}$, suggesting that cardiac growth retardation was activated in mutant hearts caused by GR dysfunction and that abnormalities in cardiac morphology were not observed in mutant neonatal hearts, implying its critical physiological role. In addition, these findings indicate the emergence of abnormal morphology and reduced cardiac systolic function in mutant adult hearts. Later, the expression of inflammatory genes was increased in mutant hearts, likely as a compensatory mechanism or possibly a subsequent suppression of glucocorticoid effects.

It has been demonstrated that HMGB1 was dispensable in heart using MHC-Cre. Therefore, we constructed cardiac-specific HMGB1 knockout mice using Ckmm-Cre $\mathrm{Hmgb1}^{\mathrm{fl} / \mathrm{fl}}$, which did not show a significant phenotype (Fig. S3). Because the onset of Ckmm-Cre, Myh6-Cre and cTnT-Cre work at different times, we speculate that this difference in Cre expression timing might account for the different phenotypes observed in these cardiac-specific knockout mice ${ }^{31}$. Therefore, caution should be exercised when interpreting the results of different conditional knockout mice. 


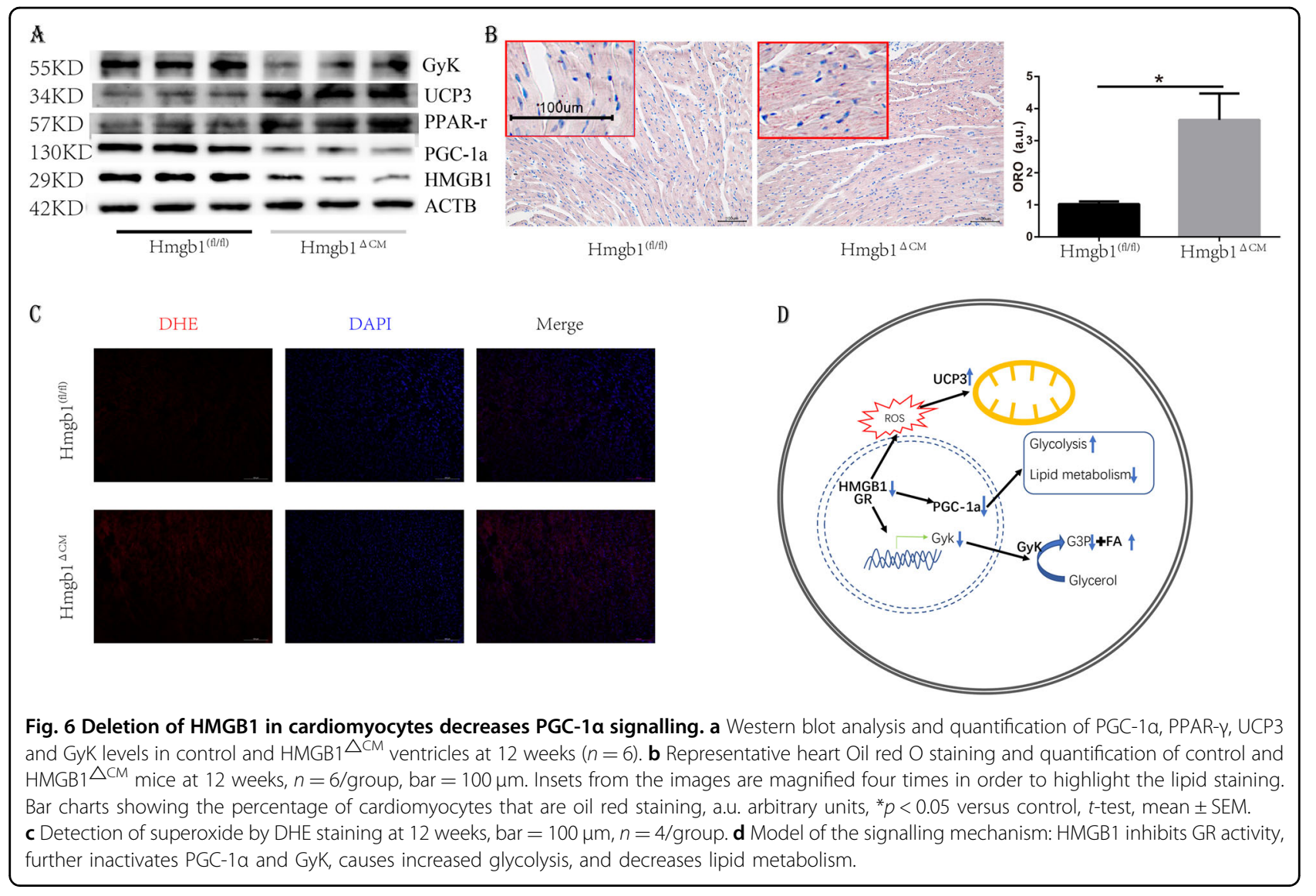

Taken together, our results demonstrate that HMGB1 is required for maintaining normal cardiac morphology and function. Loss of HMGB1 in cardiomyocytes altered glucocorticoid effects, impaired metabolic processes, and eventually resulted in small heart size and heart failure.

\section{Materials and methods \\ Animals}

$\mathrm{Hmgb1}^{\mathrm{f} / \mathrm{fl}}$ mice, in which the Hmgb1 $1^{\text {loxP }}$ allele was created by inserting loxP sites within intron 1 and intron 4 flanking exons 2-4 of Hmgb1, were kindly gifted by Prof. Taniguchi ${ }^{7}$. Mice with Cre recombinase driven by the rat cTnT promoter were kindly granted by Jiao et al. ${ }^{11}$. The mice were interbred to generate cardiac-specific HMGB1 knockout mice $\left(\mathrm{Hmgb}^{\triangle \mathrm{CM}}, \mathrm{cTnT}^{\mathrm{Cre} /+}-\mathrm{Hmgb1}^{\mathrm{fl} / \mathrm{fl}}\right)$. The control mice used in this study were $\mathrm{Hmgbl}^{\mathrm{fl} / \mathrm{fl}}$ mice without the introduction of Cre recombinase. Mice used for experiments were confirmed to be the desired genotype by standard genotyping techniques and used at the age of 1 day and 12 weeks. All mice were developed on a C57BL/6 genetic background. The sample size was ten for each group of different age or genotype, among which none was excluded. No randomization was used. The researchers were not blinded to the experiments. Animal protocols were approved by the Institutional Animal Care and Use Committee (IACUC protocol no. 2017-0007).

\section{Total RNA extraction and quantitative reverse transcriptase polymerase chain reaction}

Total RNA was extracted from heart tissue using TRIzol (Invitrogen Corp.). mRNA for HMGB1 was quantified in triplicate by SYBR Green quantitative RT-PCR. The PCR mixture was prepared using SYBR Green Master Mix (TaKaRa, Dalian, China), and the primers used are listed in Supplementary Table S1.

\section{mRNA library construction and RNA-sequencing}

Oligo(dT)-attached magnetic beads were used to purified mRNA. Purified mRNA was fragmented into small pieces with fragment buffer. Then first-strand cDNA was generated using random hexamer-primed reverse transcription, followed by a second-strand cDNA synthesis. The cDNA fragments were amplified by PCR, and products were purified by Ampure XP Beads, and then dissolved in EB solution. The double-stranded PCR products were heated, denatured, and circularized by the splint oligo sequence to get the final library. The final library was amplified with phi29 to make DNA nanoball (DNB), 
which were loaded into the patterned nanoarray and single-end 50 bases reads were generated on a BGIseq500 platform (BGI-Shenzhen, China) ${ }^{32}$.

RNA-sequencing was used to transcriptome profiling ${ }^{33}$. Bowtie was used for aligning short DNA sequence reads to large genomes ${ }^{34}$. And we used RSEM for quantifying transcript abundances from the RNA-Seq data ${ }^{35}$. The differential expression genes (DEGs) were calculated using NOISeq ${ }^{36}$, and the differential expression probability more than 0.6 was considered significant. Then we analysed biological process of the DEGs using DAVID 6.8 (https://david.ncifcrf.gov/). The raw data of RNASequencing were deposited at http://www.ncbi.nlm.nih. gov/bioproject/656926.

\section{Western blot analysis}

Myocardial protein extraction and western blotting were performed as previously described ${ }^{37}$, using antibodies against the following proteins: GR (Thermo Fisher, MA1-510), HMGB1 (Abcam, ab18256), GLUT1 (CST, 12939), GLUT4 (CST, 2213), HK2 (CST, 2867), PFK-1 (Santa Cruz Biotechnology, sc-377346), LDHA (CST, 3582), PDH (CST, 3205), CPT-1B (Abcam, ab134988), GyK (Abcam, ab126599), UCP3 (Thermo Fisher, PA1055), PPAR- $\gamma$ (Abcam, ab45036) and PGC-1 $\alpha$ (CST, 2178). Horseradish peroxidase-conjugated secondary antibody was used (Jackson, AB_10015289, AB_2313567). The band intensity of proteins of interest was normalized to that of $\beta$-actin (Bioworlde, AP0060).

\section{Co-immunoprecipitation experiments}

HMGB1 or GR was captured from heart tissue using HMGB1 antibody or GR antibody. Mouse IgG antibodies (Santa Cruz Biotechnology sc-2025) and rabbit IgG antibodies (Santa Cruz Biotechnology, sc-2023) were used as a control for non-specific-binding proteins. Heart tissues were dissected, homogenized and further resuspended in lysis buffer (Beyotime P1003J). After incubation on ice and centrifugation, the supernatant was quantified by Bradford assay, and $1 \mathrm{mg}$ of lysate was immediately precleared with Protein A/G PLUS-Agarose (Santa Cruz Biotechnology, sc-2003) at $4{ }^{\circ} \mathrm{C}$ for $1 \mathrm{~h}$. The precleared lysate was then coimmunoprecipitated overnight on a rotating wheel at $4{ }^{\circ} \mathrm{C}$ with anti-HMGB1 or GR antibody covalently coupled to Protein A/G PLUS-Agarose. For the control, the same amount of protein extract was incubated with normal mouse or rabbit non-specific IgG covalently coupled with Agarose. After the last centrifugation step, protein complexes were eluted from the beads at $95^{\circ} \mathrm{C}$ for $5 \mathrm{~min}^{38}$.

\section{Chromatin immunoprecipitation}

ChIP was performed using a SimpleChIP ${ }^{\circledR}$ Enzymatic Chromatin IP Kit (Agarose Beads) (Cell Signaling
Technology). ChIP assays used the GR antibody (Thermo Fisher Scientific, MA1-510), positive control or normal IgG contained in the kit, and were performed according to the kit protocol. Primers used for qPCR analysis of ChIP experiments are listed in Table $\mathrm{S}^{15}$.

\section{Echocardiography}

Mice underwent transthoracic echocardiography using a high-frequency ultrasound system Vevo770 (VisualSonics, Toronto, Canada). During the echocardiographic study, each mouse was positioned on a heating pad to maintain normothermia and anaesthetized with isoflurane ( $3 \%$ for induction). Left ventricular structure and function were assessed, including left ventricular posterior wall end-diastolic and end-systolic thickness, left ventricular end-diastolic and end-systolic dimensions, and LVEF.

\section{Invasive haemodynamic study}

Left ventricular haemodynamics were evaluated after echocardiography, as described previously ${ }^{39,30}$. Briefly, a micromanometer (Millar 1.4F, SPR 835; Millar Instruments, Houston, USA) was inserted into the right common carotid artery and carefully advanced into the left ventricle. The micromanometer was connected to a Power Laboratory System (AD Instruments, Castle Hill, Australia) to record heart rate and left ventricular endsystolic and end-diastolic pressure.

\section{Histological examination}

Hearts were arrested after anaesthesia by ketamine and rapidly immersed in $4 \%$ neutral formaldehyde or optimal cutting temperature compound (OCT) compound. Neutral lipid accumulation in tissues were detected and quantification using oil red staining ${ }^{40}$. Specimens embedded in paraffin were sectioned at a thickness of $5 \mu \mathrm{m}$ and stained with H\&E, Sirius Red or WGA to assess myocardial morphology and cardiac collagen content, respectively. Images were analysed using Image-Pro Plus software (Media Cybernetics, Rockville, USA).

\section{Immunohistochemistry}

For immunostaining, as shown previously ${ }^{37}$, paraformaldehyde (PFA)-fixed cryostat sections were rehydrated in phosphate-buffered saline (PBS) and blocked for 30 min in PBS containing 5\% BSA and 2\% nonimmune serum solution at room temperature. Sections were probed with HMGB1 (Abcam, ab18256), Ki-67 (Abcam, ab16667) and cTnT (BD Biosciences, 564767) (1:150 dilution) in blocking buffer overnight at $4{ }^{\circ} \mathrm{C}$. After incubation, sections were washed three times with PBS and incubated with goat anti-rabbit or mouse IgG antibody (1:200; Invitrogen, A32732 or A32723) diluted in blocking buffer at room temperature for $2 \mathrm{~h}$. Quantitative analysis of the positive areas was performed using ImageJ software. 


\section{TEM examination}

Briefly, freshly dissected left ventricular tissue was fixed with $2.5 \%$ glutaraldehyde ( $\mathrm{pH}$ 7.4) for at least $2 \mathrm{~h}$. After three washes in $0.1 \mathrm{M}$ phosphate buffer followed by fixation with $1 \%$ osmium tetroxide, specimens were dehydrated in ethanol. Ultrathin sections $(50-60 \mathrm{~nm})$ were cut on an ultramicrotome (Leica, Wetzlar, Germany) and stained with 3\% uranyl acetate and lead citrate. Images were acquired with a CM-120 microscope (Philips, Amsterdam, the Netherlands).

\section{Positron emission tomography (PET)/computed tomography $(\mathrm{CT})$ imaging}

For micro-PET/CT analysis, mice that had fasted overnight were anaesthetized with $5 \%$ isoflurane and intravenously injected with fluorodeoxyglucose (18F-FDG) prior to imaging; mice were scanned $45 \mathrm{~min}$ later (Inveon mPET/ CT; Siemens, Knoxville, TN, USA). Image quality was improved by micro-CT anatomical alignment. Data were reconstructed with the three-dimensional ordered subset expectation maximization algorithm. The maximum standard uptake value (SUVmax) was determined as a measure of cardiac glucose uptake capacity, which was normalized to the body weight of mice and total injection dosage.

\section{Statistical analysis}

Continuous variables are presented as the mean \pm standard error of the mean. Intergroup differences were assessed by Student's $t$-test or rank-sum test when nonnormal distribution via STATA software 12.0. A $p$ value of $<0.05$ was regarded as significant.

\section{Acknowledgements}

The work has been was jointly supported by the National Natural Science Foundation of China (Grant Nos. 81670355, 81800376, 81970375, 81600294 and 81521001$)$. $\mathrm{Hmgb}^{\mathrm{fl} / \mathrm{fl}}$ mice were kindly gifted by Prof. Taniguchi. Mice with Cre recombinase driven by the rat cardiac Troponin T (cTnT) promoter were kindly granted by Jiao Kai. We appreciate for their generosity.

\section{Author details \\ ${ }^{1}$ Department of Endocrinology and Metabolism, Fudan Institute of Metabolic Diseases, Zhongshan Hospital, Fudan University, Shanghai, China. ²Department of General Practice, Zhongshan Hospital, Shanghai Medical College of Fudan University, Shanghai, China. ${ }^{3}$ Shanghai Institute of Cardiovascular Diseases, Shanghai Clinical Bioinformatics Research Institute, Zhongshan Hospital, Shanghai Medical College of Fudan University, Shanghai, China}

\section{Conflict of interest}

The authors declare that they have no conflict of interest.

\section{Publisher's note}

Springer Nature remains neutral with regard to jurisdictional claims in published maps and institutional affiliations.

The online version of this article (https://doi.org/10.1038/s41420-020-00340-9) contains supplementary material, which is available to authorized users.

Received: 10 July 2020 Revised: 13 September 2020 Accepted: 24 September 2020

Published online: 20 October 2020

\section{References}

1. Chen, Z. et al. Cardiomyocyte-restricted low density lipoprotein receptorrelated protein 6 (LRP6) deletion leads to lethal dilated cardiomyopathy partly through Drp1 signaling. Theranostics 8, 627 (2018).

2. Hock, R., Furusawa, T., Ueda, T. \& Bustin, M. HMG chromosomal proteins in development and disease. Trends Cell Biol. 17, 72 (2007).

3. Ueda, T. \& Yoshida, M. HMGB proteins and transcriptional regulation. Biochim. Biophys. Acta 1799, 114 (2010).

4. Lange, S. S. \& Vasquez, K. M. HMGB1: the jack-of-all-trades protein is a master DNA repair mechanic. Mol. Carcinogen 48, 571 (2009).

5. Funayama, A. et al. Cardiac nuclear high mobility group box 1 prevents the development of cardiac hypertrophy and heart failure. Cardiovasc. Res. 99, 657 (2013).

6. Huebener, P. et al. High-mobility group box 1 is dispensable for autophagy, mitochondrial quality control, and organ function in vivo. Cell Metab. 19, 539 (2014).

7. Yanai, $\mathrm{H}$. et al. Conditional ablation of HMGB1 in mice reveals its protective function against endotoxemia and bacterial infection. Proc. Natl Acad. Sci. USA 110, 20699 (2013).

8. Calogero, S. et al. The lack of chromosomal protein Hmg1 does not disrupt cell growth but causes lethal hypoglycaemia in newborn mice. Nat. Genet. 22, 276 (1999).

9. Oka, T. Cardiac-specific deletion of Gata4 reveals its requirement for hypertrophy, compensation, and myocyte viability. Circ. Res. 98, 837 (2006).

10. Wu, B. et al. Inducible cardiomyocyte-specific gene disruption directed by the rat Tnnt2 promoter in the mouse. Genesis 48, 63-72 (2009).

11. Jiao, K. et al. An essential role of Bmp4 in the atrioventricular septation of the mouse heart. Genes Dev. 17, 2362 (2003).

12. Agah, R. et al. Gene recombination in postmitotic cells. Targeted expression of Cre recombinase provokes cardiac-restricted, site-specific rearrangement in adult ventricular muscle in vivo. J. Clin. Invest. 100, 169 (1997).

13. Puthanveetil, P. et al. Cardiac glycogen accumulation after dexamethasone is regulated by AMPK. Am J. Physiol. Heart Circ. Physiol. 295, H1753-62 (2008).

14. Agresti, A., Scaffidi, P., Riva, A., Caiolfa, V. R. \& Bianchi, M. E. GR and HMGB1 interact only within chromatin and influence each other's residence time. Mol. Cell 18, 109 (2005).

15. Love, M. I. et al. Role of the chromatin landscape and sequence in determining cell type-specific genomic glucocorticoid receptor binding and gene regulation. Nucleic Acids Res. 45, 1805 (2017).

16. Severinova, E. et al. Glucocorticoid receptor-binding and transcriptome signature in cardiomyocytes. J. Am. Heart Assoc. 8 (2019).

17. Rog-Zielinska, E. A. et al. Glucocorticoids promote structural and functional maturation of foetal cardiomyocytes: a role for PGC-1alpha. Cell Death Differ. 22, 1106 (2015)

18. Lehman, J. J. et al. The transcriptional coactivator PGC-1a is essential for maximal and efficient cardiac mitochondrial fatty acid oxidation and lipid homeostasis. Am J. Physiol. Heart Circ. Physiol. 295, H185 (2008).

19. Palomer, $\mathrm{X}$. et al. TNF-a reduces PGC-1a expression through NF-KB and p38 MAPK leading to increased glucose oxidation in a human cardiac cell model. Cardiovasc Res. 81, 703-712 (2009).

20. Lopaschuk, G. D., Ussher, J. R., Folmes, C. D. L., Jaswal, J. S. \& Stanley, W. C. Myocardial fatty acid metabolism in health and disease. Phsiol. Rev. 90, 207 (2010).

21. Hibuse, $T$. et al. The heart requires glycerol as an energy substrate through aquaporin 7, a glycerol facilitator. Cardiovasc. Res. 83, 34-41 (2009).

22. Mazzucotelli, A. et al. The transcriptional coactivator peroxisome proliferator activated receptor (PPAR) coactivator-1 and the nuclear receptor PPAR control the expression of glycerol kinase and metabolism genes independently of PPAR activation in human white adipocytes. Diabetes 56, 2467 (2007).

23. Huq, A. H., Lovell, R. S., Ou, C. N., Beaudet, A. L. \& Craigen, W. J. X-linked glycerol kinase deficiency in the mouse leads to growth retardation, altered fat metabolism, autonomous glucocorticoid secretion and neonatal death. Hum. Mol. Genet. 6, 1803 (1997).

24. Gaskell, H. et al. Ablation of Hmgb1 in intestinal epithelial cells causes intestinal lipid accumulation and reduces NASH in mice. Hepatol. Commun. (2019).

25. Huebener, P. et al. High-mobility group box 1 is dispensable for autophagy, mitochondrial quality control, and organ function in vivo. Cell Metab. 19, 539 (2014).

26. Tang, D. et al. High-mobility group box 1 is essential for mitochondrial quality control. Cell Metab. 13, 701-711 (2011). 
27. Lange, S. S., Mitchell, D. L. \& Vasquez, K. M. High mobility group protein B1 enhances DNA repair and chromatin modification after DNA damage. Proc. Natl Acad. Sci. USA 105, 10320 (2008)

28. Tang, D. et al. High-mobility group box 1 is essential for mitochondrial quality control. Cell Metab. 13, 701 (2011).

29. Ito, $H$. et al. HMGB1 facilitates repair of mitochondrial DNA damage and extends the lifespan of mutant ataxin-1 knock-in mice. EMBO Mol. Med. 7, 78 (2015).

30. Zhang, L. et al. Extracellular high-mobility group box 1 mediates pressure overload-induced cardiac hypertrophy and heart failure. J. Cell Mol. Med. 20, 459 (2016).

31. Fang, X., Wang, H., An, P., Min, J. \& Wang, F. Cardiomyocyte-specific deletion of ferroportin using MCK-Cre has no apparent effect on cardiac iron homeostasis. Int. J. Cardiol. 201, 90 (2015).

32. Yang, Y. M. et al. Hyaluronan synthase 2-mediated hyaluronan production mediates Notch1 activation and liver fibrosis. Sci. Transl. Med. 11 (2019).

33. Wang, Z., Gerstein, M. \& Snyder, M. RNA-Seq: a revolutionary tool for transcriptomics. Nat. Rev. Genet. 10, 57 (2009).
34. Langmead, B., Trapnell, C., Pop, M. \& Salzberg, S. L. Ultrafast and memoryefficient alignment of short DNA sequences to the human genome. Genome Biol. 10, R25 (2009).

35. Li, B. \& Dewey, C. N. RSEM: accurate transcript quantification from RNA-Seq data with or without a reference genome. BMC Bioinformatics 12, 323 (2011).

36. Tarazona, S., Garcia-Alcalde, F., Dopazo, J., Ferrer, A. \& Conesa, A. Differential expression in RNA-seq: a matter of depth. Genome Res. 21, 2213 (2011).

37. Liu, M. et al. Simvastatin suppresses vascular inflammation and atherosclerosis in ApoE(-/-) mice by downregulating the HMGB1-RAGE axis. Acta Pharmacol. Sin. 34, 830 (2013).

38. Di Francesco, L. et al. Visualization of human karyopherin beta-1/importin beta-1 interactions with protein partners in mitotic cells by coimmunoprecipitation and proximity ligation assays. Sci. Rep. 8 (2018).

39. You, J. et al. Differential cardiac hypertrophy and signaling pathways in pressure versus volume overload. Am. J. Physiol. Heart Circ. Physiol. 212 (2017)

40. Andrés-Manzano, M. J., Andrés, V. \& Dorado, B. Oil Red $O$ and hematoxylin and eosin staining for quantification of atherosclerosis burden in mouse aorta and aortic root. Methods Mol. Biol. 1339, 85 (2015). 